\title{
Solid-Phase Synthesis and Catalytic Screening of Polystyrene Supported Diphosphines
}

\author{
M. C. Samuels ${ }^{1}$ F. J. L. Heutz ${ }^{1}$ A. Grabulosa ${ }^{2}$ P. C. J. Kamer ${ }^{1}$
}

Published online: 25 August 2016

(c) The Author(s) 2016. This article is published with open access at Springerlink.com

\begin{abstract}
An efficient modular method towards the synthesis of a library of polystyrene supported diphosphine ligands by combining solid-phase synthesis with rational ligand design has been developed. These supported ligands, obtained in quantitative yield, were efficiently and effectively screened in Rh-catalysed asymmetric hydrogenation of several benchmark substrates.
\end{abstract}

Keywords Solid-phase synthesis - Asymmetric catalysis · Combinatorial chemistry $\cdot P$ ligands $\cdot$ Catalyst immobilisation

\section{Introduction}

Homogeneous catalysis plays an increasingly important role in chemical synthesis, both in the production of finechemicals and in the bulk industry [1-3]. Notwithstanding its huge success, there is still a pressing need for novel highly active, selective, stable and reusable catalysts of which only a handful exist despite decades of research. In particular ligands which are active in a wide range of reactions, the so-called privileged ligands, are lacking

Electronic Supplementary Material The online version of this article (doi:10.1007/s11244-016-0700-1) contains supplementary material, which is available to authorized users.

P. C. J. Kamer

pcjk@st-andrews.ac.uk

1 School of Chemistry, University of St Andrews, St. Andrews, Fife KY16 9ST, UK

2 Departament de Química Inorgànica i Orgànica, Secció de Química Inorgànica, Universitat de Barcelona, Martí i Franquès, 1-11, 08028 Barcelona, Spain
$[4,5]$. The electronic and steric properties of the ligands often have a pronounced influence on the activity, stability and selectivity of a catalyst [6-8]. Consequently ligand design has evolved as a powerful tool in the development of superior catalysts. Despite considerable progress in organometallic chemistry in the last few decades, it is often not possible to rationally design ligands and thus the development of new catalysts, especially in industry, often relies on trial-and-error [9-11]. This in turn necessitates the fast synthesis and screening of large families of ligands [11-17]. Systems based on bidentate phosphorus ligands have been shown to be highly successful in asymmetric transition-metal catalysis [18, 19], but efficient combinatorial methodologies to facilitate the synthesis and screening of vast libraries of these type of ligands are still lacking. This is mainly due to laborious (and low yielding) work-up procedures of these type of compounds and their sensitivity towards moisture and air [20].

A major tool in combinatorial chemistry to generate large compound libraries is solid-phase synthesis (SPS) [21-23]. The main advantage of combining SPS with ligand synthesis is the ease of purification, often by a simple filtration, decantation or centrifugation of the products after each reaction step. As a consequence large excesses of reagents can be used to drive reactions to completion [24]. The facile work-up makes SPS very suitable for automated equipment facilitating high throughput synthesis. Having a solid-supported catalyst also has advantages during the actual catalytic screening as it can greatly simplify the recovery of the active catalyst. Moreover it can potentially allow for the recycling of the supported catalyst which is normally problematic in homogeneously catalysed reactions [25]. The immobilisation of individual ligands and catalysts on polymeric 
supports has been well investigated [26-29], but combinatorial solid-phase synthesis of large libraries of bidentate phosphorus ligands is rare. There have been several accounts of SPS of bidentate phosphorus ligands which are mainly aminophosphine-based structures [30-34]. Recently we have reported a modular approach for recyclable supported diphosphine ligands on $\mathrm{Janda}_{\mathrm{Jel}}{ }^{\mathrm{TM}}-\mathrm{Cl}$ and Merrifield resin [35]. These supported diphosphine ligands could be very readily recycled and even related phosphinephosphite systems showed remarkable recycling efficiency [36]. Following up on these promising and versatile recycling properties we set out to create more structural diversity in our ligand libraries. Using a similar methodology we now report on an efficient combinatorial solidphase synthetic approach for libraries of highly modular bulky diphosphine ligands on polystyrene resin, which is a widely used resin in SPS.

Using polystyrene as the base structure offers several advantages as the backbone introduces a phenyl group directly on the first phosphine moiety. This makes these immobilised ligands more comparable to their solutionphase analogues than the previously reported benzyl functionality containing ligands supported on Janda$\mathrm{Jel}^{\mathrm{TM}}-\mathrm{Cl}$ and Merrifield resin. The actual support can also have an influence on the catalytic results and thus it will be interesting to investigate these support-effects in catalysis. Structural diversity was obtained by introducing several bridge structures between the two phosphine moieties using cyclic sulfates as electrophile and by varying the $\mathrm{R}^{2}$-group on the second phosphine moiety. The resulting library of seven ligands (Table 1, 9a-g) was obtained in very high yield and purity and with minimal work-up using this efficient solid-phase synthetic protocol.

Table 1 Supported diphosphine ligands 9a-g

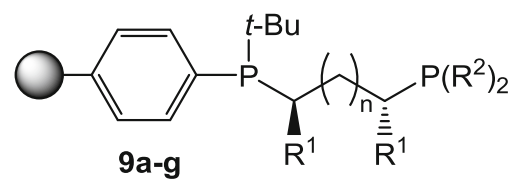

\begin{tabular}{llll}
\hline Ligand & $\mathrm{R}^{1}$ & $\mathrm{n}$ & $\mathrm{R}^{2}$ \\
\hline 9a & Hydrogen & 1 & Phenyl \\
9b & Hydrogen & 2 & Phenyl \\
9c & Methyl & $1(R \mathrm{c}, R \mathrm{c})$ & Phenyl \\
9d & Methyl & $2(R \mathrm{c}, R \mathrm{c})$ & Phenyl \\
9e & Methyl & $2(S \mathrm{c}, S \mathrm{c})$ & Phenyl \\
9f & Ethyl & $2(S \mathrm{c}, S \mathrm{c})$ & Phenyl \\
9g & Methyl & $2(R \mathrm{c}, R \mathrm{c})$ & $o$-Tolyl \\
\hline
\end{tabular}

\section{Results and Discussion}

The synthesis of the polystyrene supported diphosphine ligands could be divided into three main steps, i.e. the synthesis of the supported secondary phosphine, the addition of the ligand backbone and the incorporation of the second phosphine moiety. This is analogous to our reported work based on chloromethyl functionalised resins [35], however, to translate this to the commercially available polystyrene- $\mathrm{Br}$ resin, a new methodology had to be developed for the synthesis of the supported first phosphine moiety.

The synthesis of the secondary phosphine was first attempted in the most direct way by reacting lithiated polystyrene [37] with tert-butyldichlorophosphine followed by a reduction of the chloride. However, following this route the formation of side products was observed, which could not be removed as these side products were bound to the support. The side-product was possibly crosslinked phosphine formed by reaction of $t-\mathrm{BuPCl}_{2}$ with two aryllithiums of the resin. In a second approach, the lithiated polystyrene was reacted with tert-butylchloro$N, N$-diethylphosphinous amide [38] and transformation of the obtained supported phosphinous amide to the supported chlorophosphine was then carried out. Unfortunately, subsequent reduction to the desired supported secondary phosphine led to the same side products in this route as well.

Therefore, a more unconventional approach avoiding working with supported chlorophosphine intermediates was explored to obtain synthon $\mathbf{6}$ in the highest purity possible using tert-butylchloroethylphosphinite $\mathbf{1}$ (see Scheme 1). This results in an ethoxy-group on the immobilised phosphorus as second leaving group instead of the diethylamine-group, which could be directly reduced to the secondary phosphine.

For the synthesis of reagent $\mathbf{1}$, commercially available tert-butyldichlorophosphine in toluene was reacted with a small excess (1.1-1.2 equivalents) of freshly prepared sodium ethoxide [39] at $0{ }^{\circ} \mathrm{C}$ (Scheme 1). This resulted in a mixture of chloroethoxy-tert-butylphosphine $\mathbf{1}$ with a small amount of diethyl tert-butylphosphonite 2 (5-10\%, Fig. 1, step I) [40]. Purification of $\mathbf{1}$ was attempted but proved unsuccessful as even evaporation of the solvent under reduced pressure resulted in decomposition products with multiple ${ }^{31} \mathrm{P}$ NMR resonances between $40-90 \mathrm{ppm}$.

In situ protection of the product mixture with $\mathrm{BH}_{3} \cdot \mathrm{SMe}_{2}$ to prevent decomposition during purification was carried out, but this did not prevent the formation of some side products $(10 \% ; \delta=55-66 \mathrm{ppm})$ upon evaporation of the solvent. Unfortunately, due to the borane-group, the compound became unreactive in the reaction with lithiated 


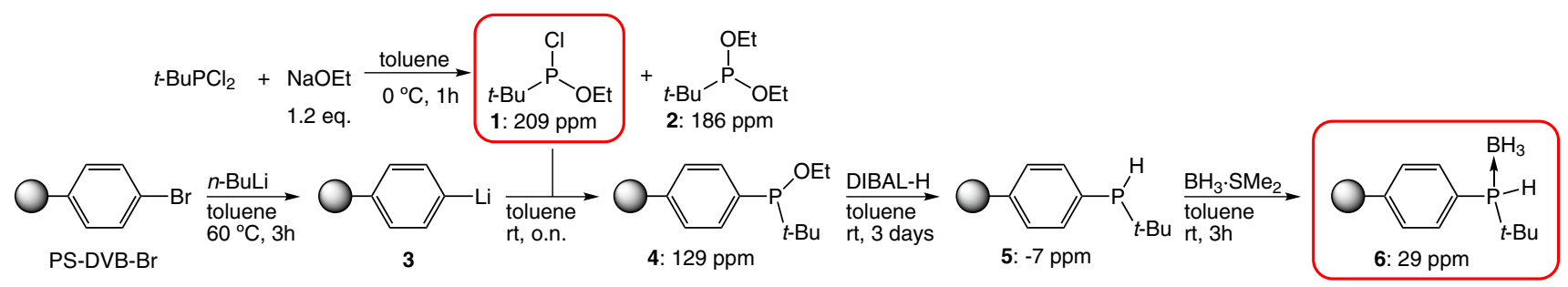

Scheme 1 Synthesis of synthon 6 via chloro-phosphinite 1

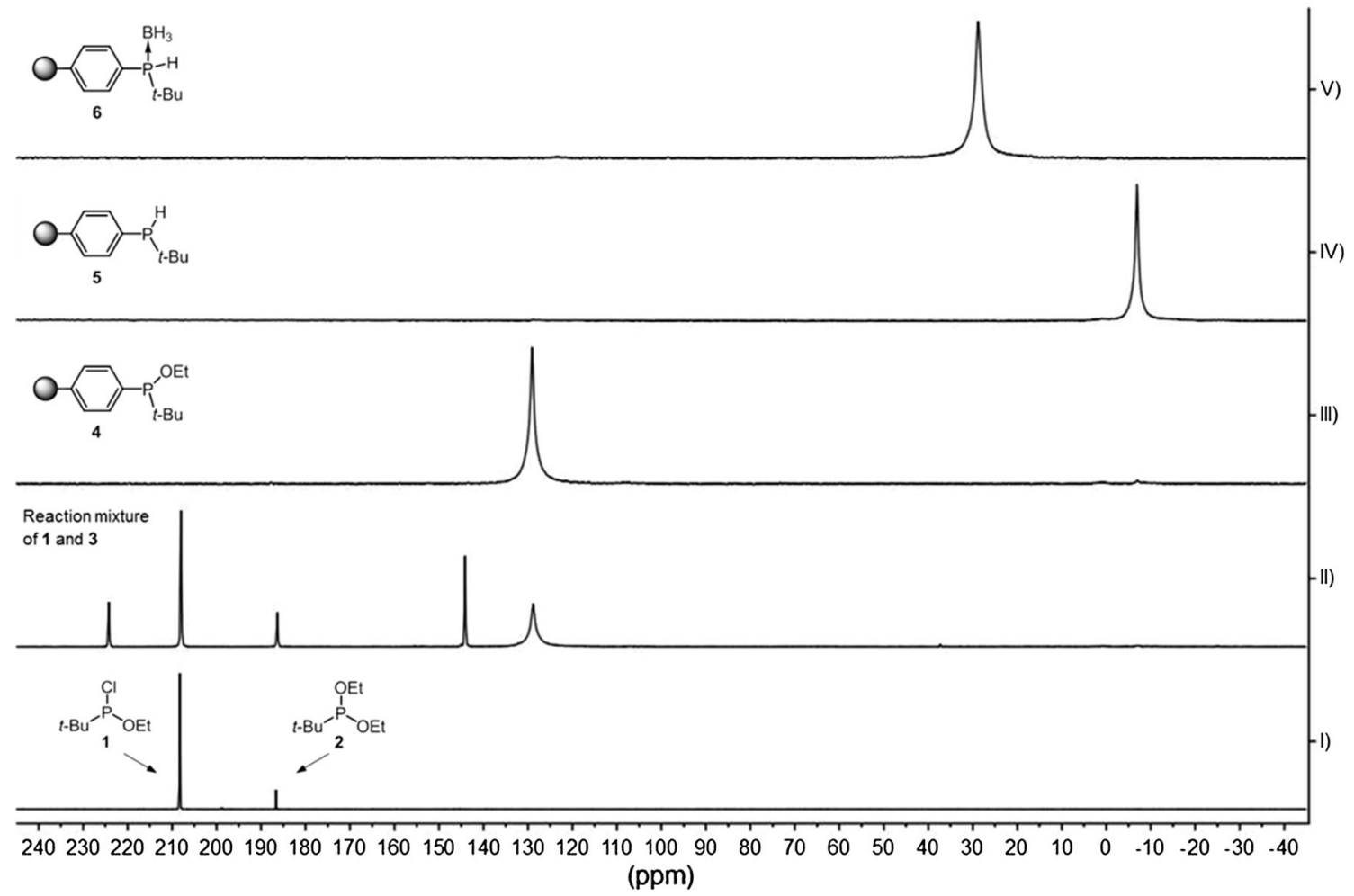

Fig. 1 Solid-phase synthesis of immobilised phosphine 6 monitored by gel-phase ${ }^{31} \mathrm{P}$ NMR

polystyrene $\mathbf{3}$. Therefore, the crude reaction mixture of $\mathbf{1}$ and 2 was used directly in excess without further purification in the reaction with lithiated polystyrene 3 (Fig. 1, step II).

After addition of the reaction mixture to $\mathbf{3}$, the excess of reagent and all the other solution-phase side-products present (Fig. 1, step II) were washed away easily, yielding phosphinite $\mathbf{4}$ as sole product attached to the resin according to ${ }^{31} \mathrm{P}$ NMR spectroscopy (Fig. 1, step III). The immobilised phosphinite $\mathbf{4}$ was then protected with borane to prevent the Arbusov reaction as described by Crofts et al [40] in their studies during the synthesis of diethyl tertbutylphosphonite 2 from tert-butyldichlorophosphine and ethanol. The reactivity of the resulting phosphinite-borane in the following reduction step towards phosphine 6 was found to be very low. Very strong reducing agents such as vitride (sodium bis(2-methoxyethoxy)-aluminium hydride solution in toluene) and diisobutylaluminium hydride (DIBAL-H) were used, but no conversion was obtained, even after heating to $50{ }^{\circ} \mathrm{C}$.

In contrast, reduction of the ethoxy-group of phosphinite 4 with DIBAL prior to the protection with $\mathrm{BH}_{3} \cdot \mathrm{SMe}_{2}$ did yield the desired secondary phosphine $\mathbf{5}$ in more than $99 \%$ purity according to ${ }^{31} \mathrm{P}$ NMR spectroscopy (Fig. 1, step IV). Subsequent protection of phosphine 5 with $\mathrm{BH}_{3} \cdot \mathrm{SMe}_{2}$ was quantitative after which the desired synthon $\mathbf{6}$ was obtained without impurities (Fig. 1, step V). This reaction could be scaled up successfully up to $5 \mathrm{~g}$ of resin without any immobilised side product formation being observed. It has to be noted that following this method, supported $P$ stereogenic phosphine $\mathbf{6}$ can only be obtained as a racemic mixture (6, Scheme 1).

With this new methodology we were able to synthesise the ligands under mild conditions and in high purity. After 
each reaction step the work-up consisted of only a simple purification via filtration and washing of the resin, making it possible to use an excess of reagents. Each step proceeded quantitatively, as indicated by gel-phase ${ }^{31} \mathrm{P}$ NMR (Fig. 1), demonstrating the efficiency of this solid-phase synthetic approach.

The bridging structure between the two phosphine moieties was introduced by lithiation of the supported secondary phosphine $\mathbf{6}$ using lithium diisopropylamide (LDA, Scheme 2), followed by addition of a set of six cyclic sulfates (Scheme 2i-vi) analogous to our previous work [35]. The ring opening of the cyclic sulfate and introduction of the ligand backbone takes place with full inversion at one of the stereogenic centres [41]. All six cyclic sulfates reacted readily with immobilised lithium phosphide $\mathbf{7}$ and immobilised sulfates $\mathbf{8 a}-\mathbf{f}$ were obtained in full conversion as confirmed by ${ }^{31} \mathrm{P}$ NMR (Fig. 2, step II).

The last step was the reaction of the supported sulfates with an excess (10-15 eq.) of different lithium phosphides to obtain the desired supported diphosphine ligands 9ag. The reaction times varied depending on the sulfate and the secondary lithium phosphide used. Once again, the excess of secondary lithium phosphide and other side products formed were easily washed away once full substitution was confirmed by ${ }^{31} \mathrm{P}$ NMR (Fig. 2, step III). Diphosphines $9 \mathbf{9}, \mathbf{b}, \mathbf{d}$ and $\mathbf{e}$ could be obtained after leaving the reaction overnight at room temperature; $9 \mathbf{c}$ and $\mathbf{f}$ were complete after 3 days and to obtain $\mathbf{9 g}$ with full conversion the reaction needed to be heated at $50{ }^{\circ} \mathrm{C}$ for 3 days. After additional treatment with $\mathrm{BH}_{3} \cdot \mathrm{SMe}_{2}$, borane protected diphosphines $\mathbf{9 a}-\mathbf{g}-\mathbf{B H}_{\mathbf{3}}$ were obtained.

Figure 2 depicts the representative synthesis of 9d monitored by gel-phase ${ }^{31} \mathrm{P}$ NMR. The integral ratio of the two phosphine moieties of $\mathbf{9 d}$ and $\mathbf{9 d}-\mathbf{B} \mathbf{H}_{\mathbf{3}}$ is 1:1, confirming the formation of the desired supported diphosphine ligand in high purity. The exact phosphorus loading of the supported ligands could be determined using elemental analysis (see ESM for detailed characterisation data).

\section{Catalysis}

The immobilised chiral ligands $\mathbf{9 c}-\mathbf{g}$ were employed in $\mathrm{Rh}-$ catalysed asymmetric hydrogenation of three benchmark substrates (Table 2). The ligands were deprotected by removal of their borane groups with 1,4-diazabicyclo[2.2.2] octane (DABCO, 10 eq.) overnight at $50{ }^{\circ} \mathrm{C}$ to obtain ligands $9 \mathrm{c}^{*}-\mathrm{g}^{*}$. Interestingly, the mixture of epimers due to the presence of a racemic stereogenic phosphorus atom in the first phosphine moiety in $9 \mathbf{d}^{*}$ could be observed after the deprotection as illustrated by the splitting of the signal (Fig. 2, VI). The catalysts were generated in situ by treatment of ligands $9 \mathbf{c}^{*}-\mathbf{g} *$ with $\left[\mathrm{Rh}(\mathrm{COD})_{2}\right]-$ $\mathrm{BF}_{4} \quad(\mathrm{COD}=1,5$-cyclooctadiene; 0.9 eq. $)$ in $\mathrm{CH}_{2} \mathrm{Cl}_{2}$. After $4 \mathrm{~h}$ incubation time, the resulting orange resins were subsequently washed and filtered. Full rhodium complexation could be confirmed by in situ ${ }^{31} \mathrm{P}$ NMR experiments. Next, substrate solutions of methyl $\alpha$-acetamidoacrylate (I), $\alpha$-acetamidocinnamic acid (II) and its methyl ester (III) in THF were added and the reaction vessels were transferred to an autoclave and charged to 1.2 bar hydrogen pressure. After a reaction time of $16 \mathrm{~h}$ the initially orange resins all had changed colour to dark brown. The reaction mixtures were then filtered over a small plug of silica to remove the resin beads and potentially free rhodium from the samples before analysis by chiral GC (see ESM for columns and conditions).

Table 2 shows that the activity and the selectivity of the tested ligands varied widely. This demonstrates why there is still a need for methods which allow the facile synthesis and screening of ligand libraries as small changes in ligand structure have a large effect on catalyst performance. Quantitative conversions could be achieved with all five catalysts for substrate I, whereas the enantioselectivity ranged from 4 to $61 \%$. For substrates II and III full conversion was only obtained for two of the ligands (9e and f) and selectivities up to $77 \%$ were observed.

The data indicates that ligand 9c bearing a C3 ligand backbone $(\mathrm{n}=1)$ appeared to be the least successful for

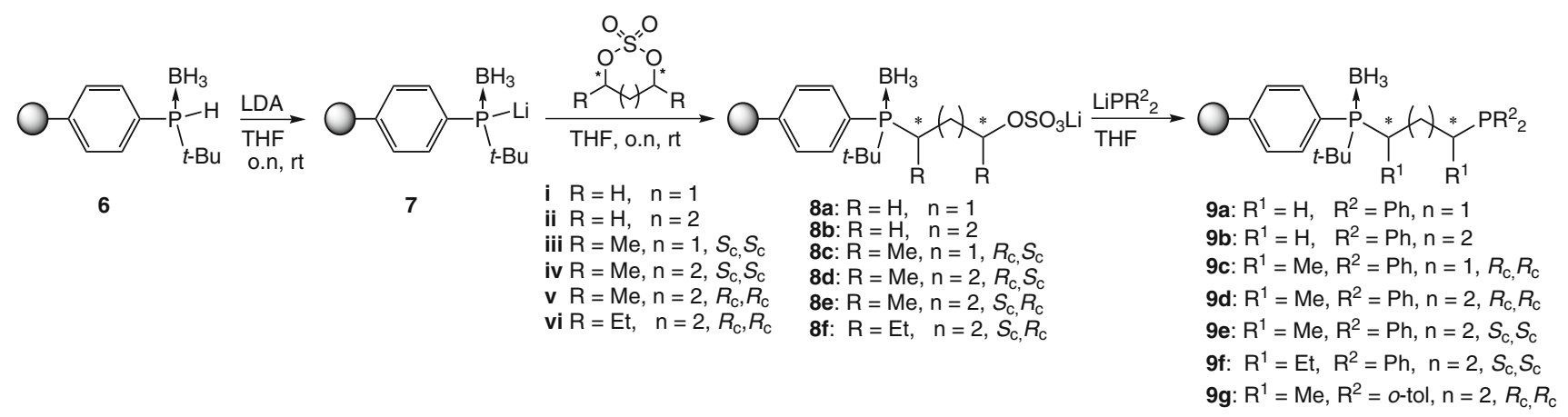

Scheme 2 Synthesis of immobilised diphosphines 9a-g 


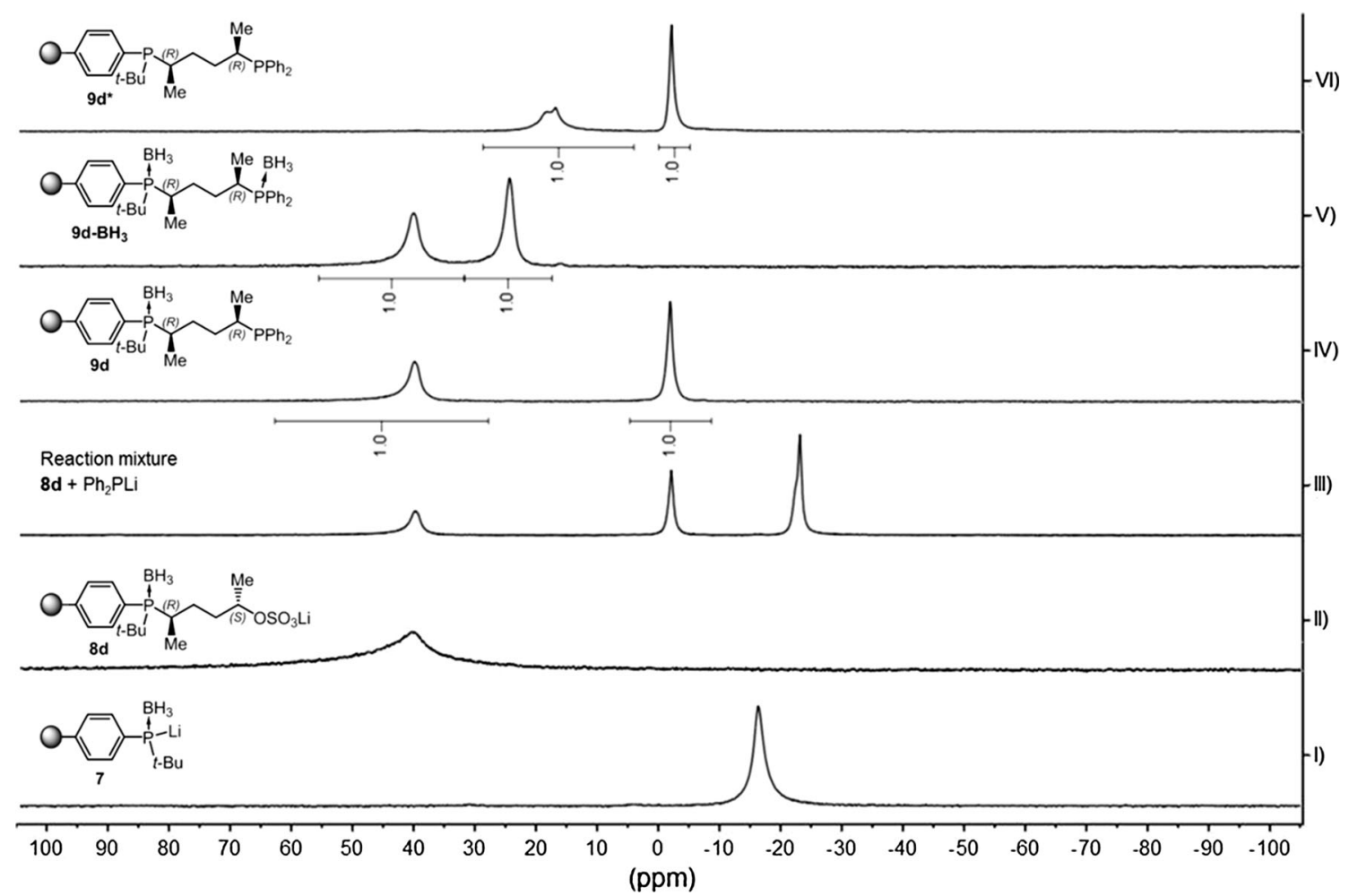

Fig. 2 Solid-phase synthesis of immobilised diphosphine 9d monitored by gel-phase ${ }^{31} \mathrm{P}$ NMR

Table 2 Results of Rh-catalyzed asymmetric hydrogenation

\begin{tabular}{|c|c|c|c|c|c|c|c|}
\hline \multirow[b]{3}{*}{ Entry } & \multirow[b]{3}{*}{ Ligand } & \multirow{2}{*}{\multicolumn{2}{|c|}{$\frac{\mathrm{NAc}_{\mathrm{H}}^{\mathrm{R}^{1}}}{\mathrm{COOR}^{2}}$}} & $\underbrace{\mathrm{COOO}^{2}}_{\mathrm{N}_{\mathrm{H}}^{\mathrm{R}}}$ & $\begin{array}{l}\text { I: } R^{1}=H, R^{2}=M e \\
\text { II: } R^{1}=P h, R^{2}=H \\
\text { III: } R^{1}=P h, R^{2}=M e\end{array}$ & & \\
\hline & & & & Substrat & & \multicolumn{2}{|c|}{ Substrate III } \\
\hline & & Conv. $^{a}$ & $e^{b}$ & Conv. $^{a}$ & $e e^{b}$ & Conv. $^{a}$ & $e^{b}$ \\
\hline 1 & $9 \mathrm{c}$ & $>99$ & $4(S)$ & $>12$ & $8(S)$ & 14.4 & $8(S)$ \\
\hline 2 & 9d & $>99$ & $23(S)$ & 53.8 & $62(S)$ & 84.5 & $46(S)$ \\
\hline 3 & $9 e$ & $>99$ & $40(R)$ & $>99$ & $55(R)$ & $>99$ & $56(R)$ \\
\hline 4 & 9f & $>99$ & $62(R)$ & $>99$ & $77(R)$ & 72.3 & $70(R)$ \\
\hline 5 & $9 \mathrm{~g}$ & $>99$ & $15(R)$ & 27 & $25(\mathrm{~S})$ & 91.5 & $7(R)$ \\
\hline 6 & BDPP & $>99^{c}$ & $40(S)^{\mathrm{c}}$ & $>99^{\mathrm{d}}$ & $93(S)^{\mathrm{d}}$ & $>99^{\mathrm{e}}$ & $72(S)^{\mathrm{e}}$ \\
\hline
\end{tabular}

Reaction conditions: $\mathrm{Rh} / \mathrm{substrate}=1: 30, p\left(\mathrm{H}_{2}\right)=1.2 \mathrm{bar}, T=25^{\circ} \mathrm{C}, t=16 \mathrm{~h}, 0.5 \mathrm{~mL}$ of THF

${ }^{a}$ Percentage conversion determined by GC

${ }^{\mathrm{b}}$ Enantiomeric excess of product determined by chiral GC (absolute configuration drawn in parenthesis)

c Data taken from Ref [42]; reaction performed at $p\left(\mathrm{H}_{2}\right)=5$ bar in $\mathrm{MeOH}$

d Data taken from Ref [43]

e Data taken from Ref [44]

these substrates and induced only very low ee's. Ligand $\mathbf{9 g}$ induced the lowest enantioselectivity of the ligands with a C4 backbone $(\mathrm{n}=2)$; apparently changing from a phenyl to ortho-tolyl group $\left(\mathrm{R}^{2}\right)$ had a detrimental effect. More interestingly, $\mathbf{9 g}$ is the only ligand that induced different absolute configurations of the hydrogenated products for the different substrates. Surprisingly for substrate I and III the $(R)$ product was observed whereas $9 \mathbf{d}$ with the same absolute configuration provides the $(S)$ product. However, for substrate II the expected opposite enantiomer $(S)$ was 
obtained again. For ligands 9d and e changing the configuration of the ligand backbone led as expected to the opposite configuration of the products in catalysis for all three of the substrates. While the employed supported ligands are used as an epimeric mixture in a 50:50 ratio, it is expected that this has only a minor influence on the catalytic selectivity. Previous work by Deerenberg et al. also has shown that for similar ligands the enantioselectivity is mainly determined by the C-chiral ligand backbone rather than the $P$-stereogenic centre $[45,46]$.

When compared to the ligand BDPP $[(S, S)-2,4-$ bis(diphenylphosphino)pentane], a homogeneous counterpart, it can be seen that for substrate II the non-supported ligand performs better and shows higher activity and selectivity. For substrate III ligand 9f exhibits enantioselectivity which is comparable to its solutionphase analogue, whereas for substrate $\mathbf{I}$ it achieves $20 \%$ higher ee. Often immobilisation of catalysts has a detrimental effect on the selectivity and activity and this nicely showcases that with our facile and modular approach we are able to develop supported ligands which perform on the same level or better than their non-supported counterparts.

\section{Conclusion}

In summary we have demonstrated a novel modular solidphase synthetic procedure for libraries of supported diphosphine ligands on polystyrene resin. Using this facile and efficient method, incorporating only a simple work-up after each step, the supported diphosphines were obtained in high purity. A new synthetic protocol has been developed for the synthesis of supported secondary phosphine $\mathbf{6}$ incorporating a bulky $t$-butyl group into the ligand structure. Subsequently, immobilised bidentate phosphine ligands $9 \mathbf{c}-\mathbf{g}$ were successfully screened in the Rh-catalyzed asymmetric hydrogenation of several benchmark substrates. The ligands displayed low to high activity and moderate selectivities, demonstrating that small changes in ligand structure can have a profound effect on the actual catalysis. The importance of trial-and-error in ligand discovery is demonstrated by these results and therefore the necessity of the development of facile combinatorial methods towards large ligand libraries. The extension to larger structural diversity will enable to combine a wider screening of the substrate scope with the anticipated good recycling performance. We are currently extending both the polystyrene supported diphosphine library and the substrate scope. Also, we are investigating the possibilities towards polystyrene supported $P$-stereogenic ligands and towards supported phosphine-phosphite/phosphinite ligands.

\section{General Experimental}

All reactions and manipulations were carried out under inert conditions using standard Schlenk techniques or in an MBraun glovebox unless stated otherwise. All glassware was dried prior to use to remove traces of water. All chemicals were obtained from commercial suppliers and used as received unless otherwise stated. Toluene was distilled from sodium, diethyl ether and THF were distilled from sodium/benzophenone and triethylamine, dichloromethane and acetonitrile were distilled from calcium hydride. Polystyrene-Br (50-100 mesh, $2.17 \mathrm{mmol} \mathrm{g}^{-1}$, $2 \%$ cross-linked DVB) was obtained from Sigma-Aldrich. Cyclic sulfates are prepared according to literature: 1,3propadiol cyclic sulfate $\mathbf{i}$ [47], 1,4-butanediol cyclic sulfate ii [48], $(2 R, 4 R)-2,4-$ pentanediol cyclic sulfate iii [41], $(2 R, 5 R)-2,5$-hexanediol cyclic sulfate iv [49], $(2 S, 5 S)-2,5-$ hexanediol cyclic sulfate $\mathbf{v}$ [49], (3R,3R)-3,6-octadiol cyclic sulfate vi [50]. See ESM for detailed synthetic procedures and characterisation of the ligands.

\subsection{General Procedure for Asymmetric Hydrogenation Experiments}

The hydrogenation experiments were performed in a stainless steel autoclave charged with an insert suitable for ten reaction vessels including Teflon mini stirring bars. In a typical experiment, a reaction vessel was charged with a deprotected resin-bound diphosphine $(2.5 \mathrm{mg}$, approximately $3.0 \mu \mathrm{mol})$ and a solution of $\left[\mathrm{Rh}(\mathrm{COD})_{2}\right] \mathrm{BF}_{4}$ (3.0 $\mu \mathrm{mol})$ in $\mathrm{CH}_{2} \mathrm{Cl}_{2}(1 \mathrm{~mL})$ and the heterogeneous mixture was allowed to stir gently for $4 \mathrm{~h}$. The supernatant solution was removed and the resulting orange resin was washed subsequently with three $1 \mathrm{~mL}$ portions of THF followed by three $1 \mathrm{~mL}$ portions of $\mathrm{Et}_{2} \mathrm{O}$. Next, a solution of substrate (30 eq.) in THF was added to the reaction vessel. Subsequently, the autoclave was purged three times with 5 bar of $\mathrm{H}_{2}$ and then pressurized to 1.2 bar. The reaction mixtures were gently stirred at $25^{\circ} \mathrm{C}$. After $16 \mathrm{~h}$, the autoclave was depressurized and the reaction mixtures were filtered over a plug of silica. Prior to GC measurements substrate II and its products were derivatized using (trimethylsilyl)diazomethane ( $2 \mathrm{M}$ in diethyl ether), in essence yielding substrate III. The conversion and the enantiomeric excess were determined by chiral GC; see supplementary information for columns and conditions.

Acknowledgments We thank the European Union (Marie Curie ITN SusPhos, Grant Agreement No. 317404) for financial support.

Open Access This article is distributed under the terms of the Creative Commons Attribution 4.0 International License (http://crea tivecommons.org/licenses/by/4.0/), which permits unrestricted use, distribution, and reproduction in any medium, provided you give 
appropriate credit to the original author(s) and the source, provide a link to the Creative Commons license, and indicate if changes were made.

\section{References}

1. van Leeuwen PWNM (2004) Homogeneous catalysis-understanding the art. Kluwer Academic, Dordrecht

2. Ojima I (2010) Catalytic asymmetric synthesis, 3rd edn. Wiley, Hoboken

3. Blaser HU, Schmidt E (2004) In: Blaser HU, Schmidt E (eds) Asymmetric catalysis on industrial scale. Wiley, Weinheim

4. Yoon TP, Jacobsen EN (2003) Science 299(5613):1691-1693

5. Zhou Q-L (ed) (2011) Privileged chiral ligands and catalysts. Wiley, Weinheim

6. Tolman CA (1977) Chem Rev 77(3):313-348

7. Deerenberg S, Schrekker HS, van Strijdonck GPF, Kamer PCJ, van Leeuwen PWNM, Fraanje J, Goubitz K (2000) J Org Chem 65(16):4810-4817

8. Gillespie JA, Dodds DL, Kamer PCJ (2010) Dalton Trans 39(11):2751-2764

9. Francis MB, Finney NS, Jacobsen EN (1996) J Am Chem Soc 118(37):8983-8984

10. Kranich R, Eis K, Geis O, Mühle S, Bats JW, Schmalz H-G (2000) Chem Eur J 6(15):2874-2894

11. Gennari C, Piarulli U (2003) Chem Rev 103(8):3071-3100

12. Reetz MT (2001) Angew Chem Int Ed 40(2):284-310

13. de Vries JG, de Vries AHM (2003) Eur J Org Chem 2003(5):799-811

14. Jäkel C, Paciello R (2006) Chem Rev 106(7):2912-2942

15. Stambuli JP, Stauffer SR, Shaughnessy KH, Hartwig JF (2001) J Am Chem Soc 123(11):2677-2678

16. Lavastre O, Bonnette F, Gallard L (2004) Curr Opin Chem Biol 8(3):311-318

17. Lefort L, Boogers JAF, de Vries AHM, de Vries JG (2004) Org Lett 6(11):1733-1735

18. Noyori R (2003) Adv Synth Catal 345(1-2):15-32

19. Knowles WS (2003) Adv Synth Catal 345(1-2):3-13

20. Goudriaan PE, van Leeuwen PWNM, Birkholz MN, Reek JNH (2008) Eur J Inorg Chem 19:2939-2958

21. Obrecht D, Villalgordo JM (1998) Introduction, basic concepts and strategies. In: Solid-supported combinatorial and parallel synthesis of small-molecular-weight compound libraries. Elsevier, Oxford, pp 1-184

22. Booth SE, Dreef-Tromp CM, Hermkens PHH, de Man JAPA, Ottenheijm HCJ (1999) Survey of solid-phase organic reactions. In: Jung G (ed) Combinatorial chemistry—synthesis, analysis, screening. Wiley, Weinheim, pp 35-76

23. Merrifield RB (1963) J Am Chem Soc 85(14):2149-2154

24. Samuels MC, Swennenhuis BHG, Kamer PCJ (2012) Solid-phase Synthesis of Ligands. In: Kamer PCJ, van Leeuwen PWNM (eds) Phosphorus(III) ligands in homogeneous catalysis: design and synthesis. Wiley, Chichester, pp 463-479
25. Cole-Hamilton DJ, Tooze RP (2006) Homogeneous catalysisadvantages and problems. In: Cole-Hamilton DJ, Tooze RP (eds) Catalyst separation, recovery and recycling-chemistry and process design. Springer, Dordrecht, pp 1-8

26. Fan Q-H, Li Y-M, Chan ASC (2002) Chem Rev 102(10): $3385-3466$

27. McNamara CA, Dixon MJ, Bradley M (2002) Chem Rev 102(10):3275-3300

28. Itsuno $S$ (ed) (2011) An overview of polymer-immobilized chiral catalysts and synthetic chiral polymers. In: Polymeric chiral catalyst design and chiral polymer synthesis. Wiley, Hoboken, pp $1-15$

29. P Barbaro, F Liguori (eds.) (2010) Heterogenized homogeneous catalysts for fine chemicals production. Catalysis by metal complexes, vol 33. Springer, Netherlands

30. Li GY, Fagan PJ, Watson PL (2001) Angew Chem Int Ed 40(6):1106-1109

31. Mansour A, Portnoy M (2001) J Chem Soc Perkin Trans 1(9): 952-954

32. Ben-Aroya BB, Portnoy M (2002) Tetrahedron 58(25): 5147-5158

33. Mansour A, Portnoy M (2003) Tetrahedron Lett 44(10): 2195-2198

34. den Heeten R, Swennenhuis BHG, van Leeuwen PWNM, de Vries JG, Kamer PCJ (2008) Angew Chem Int Ed 47(35): 6602-6605

35. Heutz FJL, Samuels MC, Kamer PCJ (2015) Catal Sci Technol 5(6):3296-3301

36. Heutz FJL, Kamer PCJ (2016) Dalton Trans. 45: 2116-2123

37. Farrall MJ, Frechet JMJ (1976) J Org Chem 41(24):3877-3882

38. Scherer OJ, Gick W (1970) Chem Ber Recl 103(1):71-75

39. Williams D, Bost RW (1936) J Chem Phys 4:251-253

40. Crofts PC, Parker DM (1970) J Chem Soc C 2:332-336

41. Fries G, Wolf J, Ilg K, Walfort B, Stalke D, Werner H (2004) Dalton Trans 12:1873-1881

42. Alame M, Pestre N, de Bellefon C (2008) Adv Synth Catal 350(6):898-908

43. MacNeil PA, Roberts NK, Bosnich B (1981) J Am Chem Soc 103(9):2273-2280

44. Bakos J, Tóth I, Heil B, Markó L (1985) J Organomet Chem 279(1-2):23-29

45. Deerenberg S, Kamer PCJ, van Leeuwen PWNM (2000) Organometallics 19(11):2065-2072

46. Deerenberg S, Pàmies O, Diéguez $\mathrm{M}$, Claver $\mathrm{C}$, Kamer PCJ, van Leeuwen PWNM (2001) J Org Chem 66(23):7626-7631

47. Samano V, Ray JA, Thompson JB, Mook RA, Jung DK, Koble CS, Martin MT, Bigham EC, Regitz CS, Feldman PL, Boros EE (1999) Org Lett 1(12):1993-1996

48. Berridge MS, Franceschini MP, Rosenfeld E, Tewson TJ (1990) J Org Chem 55(4):1211-1217

49. Bonnaventure I, Charette AB (2008) J Org Chem 73(16):6330-6340

50. Burk MJ, Feaster JE, Nugent WA, Harlow RL (1993) J Am Chem Soc 115(22):10125-10138 